\title{
Causas estructurales de los problemas de inequidad en el acceso a la salud en Chile
}

\author{
CAMILO CID ${ }^{(*)}$
}

\section{INTRODUCCIÓN}

El sector salud chileno y en particular el sistema de aseguramiento, no puede funcionar de forma razonablemente eficiente sin una regulación adecuada. Los países disponen de medidas como la afiliación obligatoria y la contribución obligatoria pareja y proporcional, para asegurar ciertos niveles de solidaridad en el sistema de salud. Esto es lo que se aplica en Chile, pero cuando la prima o contribución obligatoria está restringida por razones de solidaridad, y el mercado privado altamente desregulado, se generan incentivos a la selección de riesgos ${ }^{1}$.

La selección de riesgos es un gran problema en Chile y causa inequidad en el acceso a la salud porque promueve la segmentación de los beneficiarios, atenta contra el principio de solidaridad y es socialmente ineficiente ya que implica pérdidas de bienestar que podrían ser evitadas. Evidentemente esta selección que ocurre principalmente en el sistema Isapre, tiene efectos sobre la sustentabilidad del sistema de aseguramiento en su conjunto.

Un sistema de ajuste de riesgo en el conjunto del sistema de aseguramiento de la salud en Chile, para el conjunto de beneficios ofrecidos, que considere la particularidad del Fonasa y que las personas sin recursos encuentran solución dentro del seguro público, es un mecanis- mo de regulación que ayudará a corregir estos comportamientos socialmente indeseados y promover la solidaridad y la eficiencia en el sistema de salud. A nivel internacional es la herramienta más eficaz que se ha usado ante, menos intensos, pero similares problemas.

\section{EL PROBLEMA ES CONOCIDO: INEFICIENCIA E INEQUIDAD EN EL SISTEMA DE SALUD CHILENO}

Al estudiar los aspectos económicos más importantes de la situación del sistema de salud en Chile, -como el financiamiento en su origen y aplicación, la selección adversa o la segmentación de la demanda-, salta a la vista la necesidad de proponer un modelo que solucione los problemas de eficiencia, equidad y de falta de solidaridad existentes. Hay experiencias internacionales, especialmente en Europa, que han estado enfocadas hacia la preocupación por evitar la selección de riesgos, la expulsión de beneficiarios por los seguros y terminar con la inequidad en el acceso que ello conlleva. Países como Holanda, Alemania, Bélgica y Suiza, entre otros, han dado pasos firmes en las soluciones partiendo de un diagnóstico sistemático y serio. (van de Ven et. al., 2001, Van de Ven et al, 2007).

(*) Departamento de Estudios. Superintendencia de Salud. Mac Iver 225. Santiago. Chile. camilocid@gmail.com

1 Cuando existe selección los seguros privados ajustan las primas según el costo esperado del individuo, llegado el momento el individuo no puede pagar dicha prima y es expulsado del seguro y eventualmente del sistema de seguros (van de Ven et. al, 2001). 
Está demostrado que en un mercado competitivo de seguros de salud, sin ninguna o una pobre regulación, las primas asociadas a riesgos serán altas para las personas adultos mayores, para enfermos, mujeres en edad fértil y familias numerosas y, serán bajas para los jóvenes, los solteros y las familias pequeñas. Esto es lo que ocurre, ya no teóricamente, sino que en la realidad en el sistema Isapre en Chile.

Además, como ha sido ampliamente documentado, en Chile se aprecia una importante inequidad en el financiamiento de la salud entre los sectores público y privado y entre quintiles ricos y pobres de la población (Titelman y Uthoff, 2000, Larrañaga 2000, Superintendencia de Salud, 2006), ello facilitado por los arreglos institucionales vigentes que separa el financiamiento entre aquel que es solidario del Fonasa, constituido por el aporte fiscal y las cotizaciones de las personas de más bajos ingresos en partes casi iguales y el individual, de las Isapres, asociado al $15 \%$ a $20 \%$ más rico de la población que está afiliada a ellas y que no ejerce ninguna solidaridad en el financiamiento de la salud. Ello y otras desigualdades estudiadas por diversos autores implican importantes diferencias en el acceso a la salud (Vega, 2001, Vega et al, 2003, Subramanian et al, 2003, etc)

Por otra parte, los altos co-pagos y gasto de bolsillo de la población (33\% en promedio según Superintendencia de Salud, 2006) debilitan la necesaria responsabilidad financiera de los se- guros de salud, entendida como el grado en que los gastos de los beneficiarios se reflejan en los estados financieros de los seguros y no en los estados financieros de los individuos o de instituciones del gobierno. Que los seguros asuman mayor responsabilidad financiera es un incentivo a la contención de costos y así lo ha entendido la regulación de los países que hemos señalado antes (Van de Ven et. al. 2001 y 2007).

La obligatoriedad en el aseguramiento y las primas proporcionales al ingreso son instrumentos clásicos que los países se dan para poder promover la solidaridad en los sistemas de salud. Con ello se promueve que la prima se calcule independientemente del estado de salud de las personas y que sea igual para todos, independiente del número de personas de la familia, de los ingresos y de los riesgos de enfermar. Inicialmente, esta es la razón técnica fundamental para que la prima en Chile, al igual que en muchos otros países con sistemas de seguridad social, sea una proporción del ingreso (en este caso 7\%). No obstante, un aspecto diferencial del caso chileno son las primas en Isapres que se determinan con el $7 \%$ del ingreso como un piso y con un monto diferencial determinado por el riesgo, de tal forma que la prima promedio del sistema Isapre alcanza en promedio cerca del 10\% del ingreso y va creciendo de año en año (Superintendencia de Salud, 2006). Por otra parte, esta libertad de fijación de la prima de los planes, facilita la aplicación de la selección ${ }^{2}$.

2 Por ejemplo, gracias a esta flexibilidad, en la adecuación de precios de este año las Isapres aplicaron en promedio un alza real del $8 \%$ a los planes de salud, cuestión que fue altamente criticada por todos los sectores políticos, por la Superintendencia de salud y por cierto por los beneficiarios. Cabe señalar que la reforma dotó de un mecanismo de regulación de precios a la Superintendecia de salud, que consiste en establecimiento de una banda para la variación de los mismos (30\%). La fórmula se ocupa fundamentalmente de disminuir la varianza de los precios de planes al interior de isapres de manera de evitar el castigo extremos a los más riesgosos. No obstante, si bien las alzas iban en baja hasta 2007, el mecanismo no pudo controlar el alza desmedida de 2008. Decisión reciente Tribunal Constitucional. 


\section{LA SELECCIÓN DE RIESGOS: UNA CAUSA FUNDAMENTAL DE PROBLEMAS DE ACCESO A LA SALUD EN CHILE}

Cuando la prima está legítimamente restringida porque está asociada al ingreso, para evitar regresividad en la contribución obligatoria a la seguridad social y a pesar de la parte libre asociada al riesgo individual en el sector Isapre, surgen incentivos para la selección de riesgos. Estos elementos suelen generar incentivos a la selección, más aún si existe un entorno institucional desigual y con una regulación débil. En efecto, en el caso chileno existe una fuerte inequidad en el financiamiento, graves problemas de selección sin una fuerte regulación al respecto y un sistema de salud segmentado por ingresos y riesgos, problemas que persisten aún con la implementación de la reforma.

La selección de riesgos en Chile tiene varias facetas de ineficiencia. Primero, las Isapres están desincentivadas a responder adecuadamente a los requerimientos de los afiliados de alto riesgo. Esto provoca un problema de calidad en los tratamientos, por ejemplo, de los enfermos crónicos. Segundo, el éxito en atraer beneficiarios de menor riesgo, que logran los seguros privados, provoca segmentación del mercado, donde se cobran primas mas bajas a estos afiliados de bajo riesgo y primas más altas a los individuos de mayores riesgos (y simplemente no se generan planes con primas de cierto nivel de ingresos hacia abajo). Esto genera un problema de solidaridad en el conjunto del sistema de seguridad social porque impide la compensación de ricos hacia pobres y de sanos hacia enfermos. En Chile, además, importantes segmentos de personas afectadas por el "descreme" en el sector Isapre, que ya no están dispuestas a pagar primas altas en el sistema privado, se dirigen a Fonasa, donde podrán pagar la prima más baja posible para ellos (el 7\% de su ingreso) y obtener una cobertura razonable. El efecto financiero neto de esta situación en Fonasa, puede ser deficitario. Si es así, el pooling del seguro público deberá cubrir el déficit con recursos de los cotizantes o desde el Gobierno, lo que implica un problema de equidad, al reducirse los recursos que estaban disponibles, para el conjunto de los beneficiarios, antes del ingreso de estas personas (Blackburn et al, 2004).

Además en este marco, para las Isapres, es más rentable la selección, que mejorar la eficiencia en la producción de atención de salud que se traduzca en contención de costos. En el corto plazo, los seguros prefieren invertir sus recursos disponibles en mejorar la selección de riesgos y no en reducir costos, y por lo tanto, no invierten en mejorar la eficiencia en la atención de salud en su relación con los proveedores. Los seguros más eficientes, que aplican menos selección, pueden perder participación en el mercado respecto de los seguros más ineficientes, resultando en una pérdida de recursos para la sociedad. Por otra parte, mientras un seguro individual puede obtener ganancias de la selección y beneficiarse, para la sociedad esto es una pérdida, y resulta en una pérdida de bienestar general (Wasem, 2003).

\section{LA SALIDA TAMBIÉN ES CONOCIDA: PRIMA COMUNITARIA GLOBAL Y COMPENSACIÓN POR RIESGOS}

La manera de evitar esta situación y lograr que los subsidios de solidaridad funcionen, preservando la necesaria competencia en el sistema Isapre, es generar subsidios a las primas de los grupos de alto riesgo provenientes de los grupos de menores riesgos, mediante el uso de un modelo de ajuste de riesgos que incluya a Fonasa. Se entiende por tal, el uso de información para estimar los costos esperados en salud de un individuo o grupo de individuos, basándose en la utilización y los costos observados durante un intervalo fijo de tiempo (típicamente un año), para establecer subsidios a la prima de los grupos de alto riesgo (Ellis et al, 1996). De esta manera, el ajuste de riesgos puede neutralizar los incentivos para la selección de riesgos, en la medida que, a los 
seguros les sea cada vez más indiferente la afiliación de una u otra persona. Estas medidas son aplicables en Chile incluyendo al seguro público Fonasa $^{3}$, que es el aspecto diferencial respecto de la experiencia internacional, la adaptación al caso chileno exige tener en cuenta ello y por lo tanto asegurar el financiamiento, al menos de aquellas personas que no podrán pagar un seguro en otra parte, es decir, asegurando el subsidio público de esas personas en Fonasa.

No obstante lo anterior, está demostrado que mientras más refinado sean los ajustadores, más exactas serán las predicciones de gastos en atención de salud, que permitan disminuir los incentivos de selección (Newhouse et. al., 1989). Para Chile un modelo de ajuste de riesgo que contemple sólo edad/sexo es inadecuado, porque esto considera demasiados pocos factores o características relevantes del paciente y es capaz de predecir sólo una pequeña parte de la variación en el empleo de recursos en salud. Cuando un modelo como ese es usado para el pago de cápitas ajustadas a los seguros de salud puede favorecer la selección y aumentar los problemas de acceso. Los modelos basados en diagnósticos de morbilidad pueden distinguir mayormente las diferencias de gastos esperados simultáneamente y explicar más y mejor la variación observada en gastos de salud a través de los individuos. (Cid y Wasem, 2008).
También existe un conjunto de otras normas, que acompañan estos modelos, entre las más importantes está la afiliación abierta ${ }^{4}$ que cumple un papel complementario. Esto es un hecho en países que cuenta con sistemas de ajuste de riesgos como Alemania, Israel y Holanda y Suiza (Van de Ven et al, 2001, Van de Ven et al 2007). En Chile, en la práctica sólo Fonasa funciona con afiliación abierta, ya que debe recibir a toda persona que lo solicite, lo que sólo es sostenible por el subsidio a la oferta que realiza el Estado hacia el sistema público de salud que siempre debe atender a los beneficiarios del Fondo. Así, avanzar hacia la afiliación abierta en los seguros privados de salud y la regulación vía el ajuste de riesgo se hace aún más necesaria.

\section{LA PORTABILIDAD ES SUPERFLUA EN EL CONTEXTO CHILENO}

Por otra parte, la libertad de elección en salud, como se entiende en Chile ${ }^{5}$, no es un principio necesario si hay equidad y eficiencia con calidad en la atención de salud, países con sistemas nacionales de salud como el Reino Unido y España, entre otros, han mostrado que la integración sanitaria y el desarrollo de la atención de salud en redes para una población sujeta a un territorio de salud es la mejor alternativa en dichos casos, incluso algunas HMOs de Estados

3 Este modelo pero restringido a las GES y sus costos estimados, fue propuesto al congreso nacional por el gobierno (2003) y rechazado por la derecha durante las discusiones de la Reforma de Salud. Ha sido simulado por Ministerio de salud 2001; Vargas et.al. 2005. Finalmente por Cid y Wasem 2008 ampliado al conjunto del gasto en atenciones de salud y utilizando diagnósticos hospitalarios como ajustadores. También ha sido argumentado su uso para Chile por Wasem, 2003 y Blackburn et al, 2004.

4 Las personas pueden cambiarse de seguro de salud y este debe aceptar cualquier solicitud de afiliación. El procedimiento generalmente está sujeto a algunas normas habituales como período mínimo de contrato, sujeto a área de desempeño del seguro, períodos previos de notificación, período determinado del año en que se puede realizar el cambio, etc.

5 Tiende a asociarse a portabilidad, es decir, que los recursos públicos destinados al subsidio de los indigentes del Fonasa, se comporten como un subsidio a la demanda y puedan ser tomados por ellos y destinados a contratar un seguro privado o isapre, para cubrir un plan universal, en la práctica las GES-AUGE (Vergara, 2008). La portabilidad tiene también otra cara, que es la atención en hospitales públicos de cotizantes de Isapres de manera generalizada y no restringida como es actualmente. 
Unidos, como Kayser Permanente, han aprendido a contener costos y ser mas eficientes con este tipo de modelo.

Por lo demás la libertad de elección está limitada en todo el sistema de seguridad social de salud chileno. Es un mito que sea universal en el sistema privado, la selección de pacientes y prestaciones que opera persistentemente en tal sector, provoca que la garantía de libre elección que se pregona no sea efectiva ya que siempre existe la posibilidad de exclusión por riesgo, lo que obliga a los beneficiarios a ser cautivo de la elección de la Isapre anterior, lo mismo ocurre por nivel de renta. Lo anterior deja a esta población excluida de posibilidad de elección. Este mal no es menor si se toma en cuenta que los cautivos sólo por edad se acercan al $10 \%$ de los beneficiarios. Este porcentaje sube ostensiblemente si consideramos a los enfermos crónicos y los cotizantes de menores ingresos de las Isapres.

El argumento de la portabilidad, es decir, transformar el subsidio de Fonasa a los beneficiarios más pobres en un subsidio a la demanda, en pos de la libre elección, se vuelve vano en este contexto real. Aparte de los problemas de desfinanciamiento del sector público que podría generar, la portabilidad siempre implica subsidio a las Isapres, ya sea a través del aporte a las personas para que compre un plan en el sector privado de un valor superior al aporte y en el encuentro con el sistema de atención de salud, cuando beneficiarios de Isapres se atiendan en hospitales públicos a precios subsidiados que serían capturados por la Isapre en cuestión. El Gobierno no se puede dar el lujo de subsidiar a un sector con tamañas ineficiencias económicas como las descritas anteriormente y que no garantiza la libre elección, que sería la principal razón para dicho subsidio. Sólo el sector público, Fonasa, está en condiciones de asegurar la atención de salud razonablemente efectiva a la población más pobre.

\section{CONCLUSIÓN Y DISCUSIÓN}

Los problemas centrales que causan problemas de equidad en el acceso al sistema de salud en Chile pueden ser solucionados y la portabilidad no tiene que ver ni con las causas, ni con las soluciones de ellos.

En mercados con muchas fallas, como los de salud, se produce un "trade-off" entre equidad y eficiencia, lo que resulta ser un desafío para los gobiernos y entidades técnicas regulatorias. La redistribución de riesgos intergeneracional, desde jóvenes a viejos y desde sanos a enfermos, puede producir un mejoramiento de ambos conceptos relevantes para las políticas de salud: equidad y eficiencia. En este contexto, mientras mejor sea la calidad del ajuste de riesgos, menos intensivo será este trade-off.

Los modelos posibles son varios, pero en este análisis subyace uno en particular, cual es el de financiamiento equitativo y pagador único hacia el aseguramiento público y privado, determinación de una prima comunitaria amplia (el plan implícitamente establecido en el arancel MLE del Fonasa, por ejemplo) y ajuste de riesgo para las transferencias hacia los seguros conservando las particularidades del Fonasa como es la necesidad de subsidiar a los pobres dentro del seguro público y considerar la morbilidad existente y esperada del seguro público que mantiene y ha estado recibiendo por largos años los "malos riesgos" desde el sistema Isapre.

Por otra parte, en las evaluaciones existentes (Superintendencia de Salud, 2007), se puede apreciar que el modelo de Fondo de ajuste de riesgos inter-Isapre implementado por la reforma tiene efectos extremadamente limitados. El fondo propuesto inicialmente, pero rechazado por los grupos de interés en el Congreso asociados a la derecha, solidarizaba el financiamiento de ambos mundos público y privado pero también estaba limitado al paquete básico que son las GES-Auge.

Dado lo anterior, es necesario avanzar hacia un ajuste incorporando la morbilidad que se 
muestra muy superior que el ajuste meramente demográfico y al conjunto de beneficios más amplio posible para fijar la prima comunitaria.

Suponiendo la existencia de voluntad política de implementar este sistema que permita avanzar en las soluciones, en el campo estrictamente técnico, se debe avanzar en la generación de un conjunto básico mínimo de datos, exigible en todo el sistema de aseguramiento (Fonasa e Isapres), a nivel individual que registre de manera continua o relacionada todos los encuentros de los pacientes con el sistema a cualquier nivel de atención. Por otro lado, este registro debe ser sistemático de manera de poder explorar los modelos prospectivos reportados como generadores de mejores incentivos a la eficiencia. Además se debe poner especial atención en los registros de la atención ambulatoria y de las prescripciones farmacéuticas, para poder estimar el componente ambulatorio de la atención de salud.

Siguiendo con la visión optimista, mientras se obtiene la viabilidad política-institucional a un modelo completo y amplio como el que se propone en estas notas, se puede avanzar en políticas complementarias como la instauración de la afiliación abierta en el sistema Isapre y la implementación de un fondo de riesgo compartido para altos riesgos y altos costos, en nuestra impresión, sin demasiados inconvenientes.

Recibido: 6 junio 2008 Aprobado: 13 agosto 08

\section{REFERENCIAS}

1. BLACKBURN S., ESPINOSA C., TOKMAN M. (2004). "Alternativas para reducir la discriminación y la segmentación por riesgo en el sistema de salud chileno". Serie financiamiento para el desarrollo 152 CEPAL, Diciembre de 2004.

2. CID C., WASEM J. (tutor) (2008). "Diagnosesbased risk adjusted capitation payments for im- proving solidarity and efficiency in the chilean health care system: evaluation and comparison with a demographic model". PhD in Economics thesis. Institute for Health Care Management. University of Düisburg-Essen.

3. CID C., MUÑOZ A., SÁNCHEZ M., TEGTMEIER R. (2007). "Fondo de Ajsute de Riesgos en el Sistema Isapres". Publicado en www. supersalud.cl, Biblioteca digital.

4. ELLIS R, POPE G, IEZZONI L, AYANIAN J, BATES D, BURSTIN H, ASH A. (1996). "Diagnoses-Based Risk Adjustment for Medicare Capitation Paymentes" Health Care Financing Review, spring vol. $17 \mathrm{~N}^{\circ} 3$.

5. MINSAL "FONDO SOLIDARIO EN LA REFORMA DE SALUD CHILENA". PREPARADO POR C. CID. Departamento de Estudios Gabinete. Noviembre 2001. Documento interno.

6. NEWHOUSE J., MANNING W., KEELER E., SLOSS E. (1989). "Adjusting Capitation Rates Using Objective Health Measures and Prior Utilization" Health Care Financing Review, Spring vol $10 \mathrm{~N}^{\circ} 3$.

7. SUBRAMANIAN JV., DELAGADO I., JADUE L., VEGA J., KAWACHI I. (2003) "Income inequality and health: multilevel analysis of Chilean communities". J Epidemial Community Health 2003; 57: 844-848.

8. SUPERINTENDENCIA DE SALUD (2006) "Evaluación y monitoreo de la reforma: determinación de línea basal de equidad en el financiamiento y protección financiera". www.supersalud.cl, Biblioteca digital. Febrero de 2006.

9. TITELMAN D., UTHOFF A. (2000). "El Mercado de la salud y las reformas al financiamiento de los sistemas de salud" en Ensayos sobre el financiamiento de la seguridad social de salud. Los casos de Estados Unidos - Canadá - Argentina Chile - Colombia. Volumen I. CEPAL-Fondo de Cultura Económica, 2000

10. VAN DE VEN WYNAND P.M.M., KONSTANTIN BECK, FLORIAN BUCHNER, DOV CHERNICHOVSKY, LUCIEN GARDIOL, ALBERTO HOLLY, LEIDA M. LAMERS, ERIK SCHOKKAERT, AMIR SHMUELI, STEPHAN SPYCHER, CARINE VAN DE VOORDE, RENÉ C.J.A. VAN VLIET, JÜRGEN WASEM, IRITH ZMORA (2001). 'Risk adjustment and 
risk selection on the sickness fund insurance market in five European countries'. Version 3, 29 October 2001.

11. VAN DE VEN WYNAND P.M.M., BECK KONSTANTINE, VAN DE VOORDE CARINE, WASEM JÜRGEN. ZMORA IRIT (2007). "Risk adjustment and risk selection in Europe: 6 years later" Health Policy 83 (2007) 162-179.

12. VARGAS V., CID C., MATUS M. ET.AL. (2005). 'Una Metodología para Ajustar y Compensar las Diferencias de Riesgo entre el Sistema Público y Privado de Salud en Chile'. Ensayos Ilades/Georgetown University. www. Ilades.cl.

13. VEGA J., BEDREGAL P., JADUE L., DELAGADO I., (2003). "Equidad de género en el acceso a la atención de salud en Chile". Rev. Med. Chile, v131 n.6 Santiago jun, 2003.
14. VEGA J. (2001). "Enfermo de Pobre. Las Inequidades sociales y la salud en Chile". Revista Universitaria $\mathrm{N}^{\circ} 73 / 2001$.

15. VERGARA M. (2008). "Equidad en el acceso a la salud en Chile". Revista Chilena de Salud Pública 2008; Vol 12 (2): 156-163.

16. WASEM, J. "The Solidarity Fund in Chile on its way to implementation-some observations from an international perspective", Final Report, GTZ mission to MOH of Chile, March 2003.

Usted puede comentar éste y otros artículos publicados en la Revista Chilena de Salud Pública, enviando un correo electrónico a revistasp@med.uchile.cl 